\title{
FROM NATURE AND BASIC SCIENTIFIC RESULTS TO MODERN ENGINEERING APPLICATIONS
}

\author{
GIORA ROSENHOUSE \\ Swantech Ltd. Haifa, Israel Technion, Haifa, Israel (Retired Prof.)
}

\begin{abstract}
The motto of the paper concerning physics and nature is a quotation by Eugene Wigner: 'The miracle of the appropriateness of the language of mathematics for the formulation of the laws of physics is a wonderful gift which we neither understand nor deserve'. The first case in this paper is based on an original paper by this author, and it uses Fletcher's scale of speech intelligibility, for analysis of specific noise effect in the presence of background noise. The idea is based on the assumption that the brain uses the same scale for estimating both intelligibility and nuisance by specific noise. This notion was confirmed by experiments. The second case below is the use of the 'simplex theory', which is a mathematical model used in computer sciences, and was published originally by this author as a means for design of environmental sound barriers. The third application involves sophisticated use of specular reflections and scattering in acoustics, with application of acoustic quadratic residues in interiors acoustics, based on the mathematical theory of numbers, where prime numbers are involved. Leading mathematicians in this development are Euclid (325 BC-265 BC) and Gauss (1777-1855), who discovered in the 18th century quadratic residues. In the context of diffraction physics, the main contribution was by Röntgen (1845-1923), von Laue (1879-1960), W.H. Bragg (1862-1942), and W.L. Bragg (1890-1971). This long way towards sophisticated acoustic diffusers that turn specular reflections into a uniform diffusion needed one more leading physicist to make the breakthrough. Manfred Schroeder (1926-2009) published a seminal paper in 1975, adding the number theory to room acoustics as a legitimate part. He has proved mathematically that specific panels with a sequence of one-dimensional or two-dimensional grooves result in a diffusive-phase grating of wide band, instead of a specular reflection panel. This result was directly applicable from the theory of $\mathrm{x}$ ray diffraction. D'Antonio and Cox continued improving the results, leading to special quadratic residue diffuser (QRD) shapes. Many of the panels resemble shapes that exist in nature.

Key words: BS 4142: 2014, outdoor noise control, physical innovations, primary numbers, quadratic reduced difftusors (QRD), scattering of sound, Simplex method, Sound Barriers, squealing noise, Subjective acoustics.
\end{abstract}

\section{INTRODUCTION}

Motto 1: Leonardo da Vinci ( 1452-1519), "Those who fall in love with practice without science are like a sailor who enters a ship without a helm or a compass, and who never can be certain wither he is going", Notebooks of Leonardo da Vinci.

\subsection{The use of basic mathematics and measurements in physics and engineering}

Pure mathematical rules, and scientific measurements that may look useless, can be the keys for advanced technological applications. On the other hand, we find also such physical phenomena in nature as it is. Simply by watching with our eyes and ears what exists around us, the solutions are there. Probably, though, they are not as quantitative as we need for exact design purposes. We show by three studies how basic physical measurements can lead to applied observations.

Because of their basic nature, such rules and observations 'wait' sometimes, even thousands, hundreds of years or decades, until their published consequences are used in modern applications. 


\section{THE SUBJECTIVE ANALYSIS OF WHEEL- RAIL SQUEALING NOISE BY MODIFICATION OF THE BRITISH STANDARD 4142: 2014}

Case studies, based on acoustical measurements of the effect of signal to noise caused in industrial premises and environmental domains, can be concerned with extreme human sensitivity to certain patterns of the noise, even if its total amount remains unchanged compared with other events. The case of rail-wheel squealing noise is characterized by extremely high noise levels, above background noise, at frequencies to which people are sensitive. We refer to, say, $70-80 \mathrm{~dB}$, and up to $120 \mathrm{~dB}$, depending on the distance between the source and the observer, see Fig. 1.
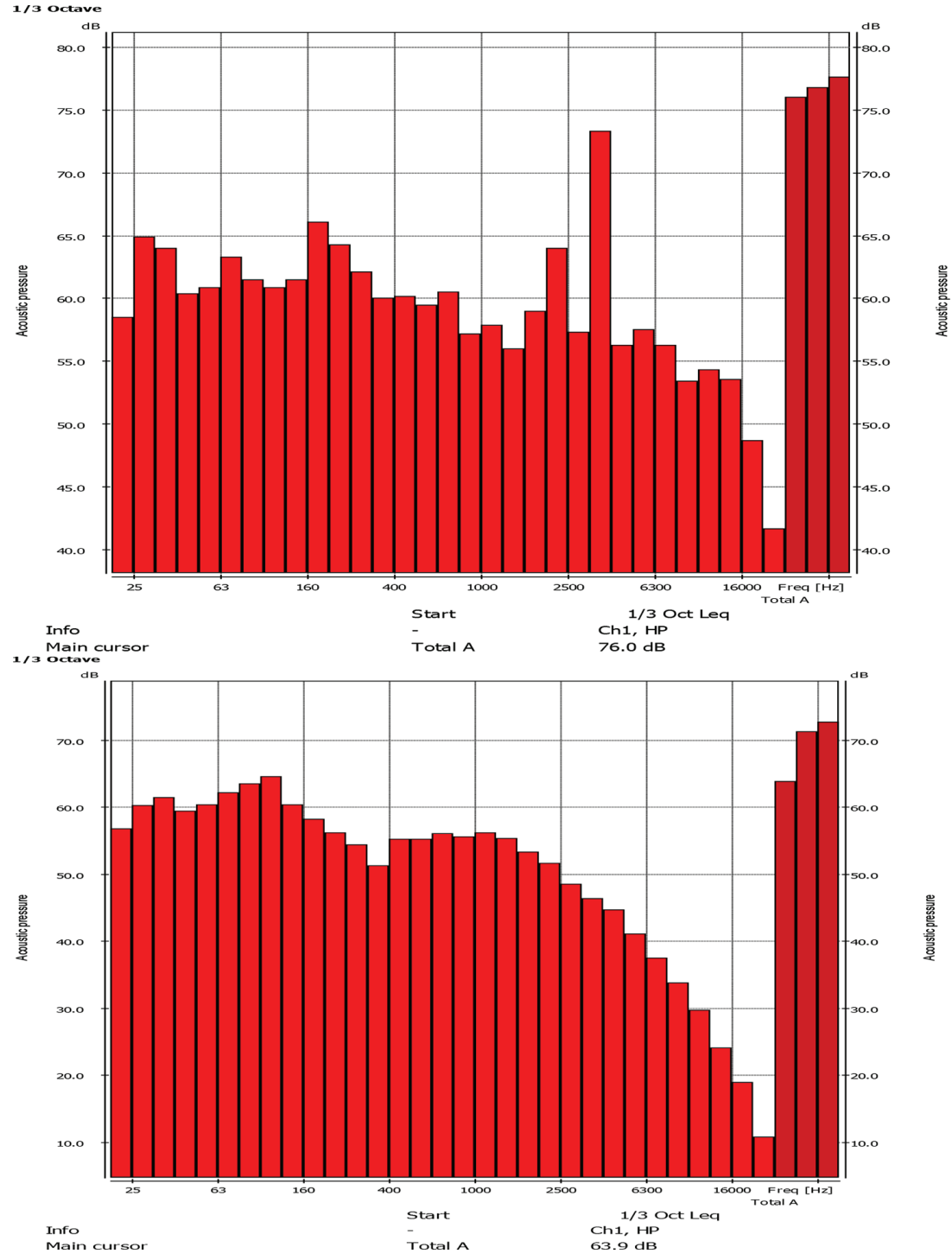

Figure 1: Spectral plots of background noise (bottom) and wheel/rail squeal (top) [4]. 
Wheel-rail squealing noise depends on geometries, such as the diameter of the wheel and the radius of the curve, mass of the wheel and type of the train, which affect the dynamic response of the system. In addition, changes in the conditions and state of the system, such as weather (rainy, dry), slippery rails, effect of the stick-slip phenomenon that yields wheel squeal curving noise, rail imperfections, joints and certain truck designs, as well as wheel rail separation and hardly worn rail, can result in impact noise. Rail and wheel roughness, material heterogeneity, curved and tangent curves can lead to rolling noise.

This large amount of noise causes, many of which can appear and disappear, makes it almost impossible to eliminate, for the long run, wheel-rail squeal noise, and much research has studied this problem since 1954 and up to-date [1-4]. Yet, during the 50 years of use, the standard BS 4142 [5] and ISO 1996 [6] have not given sufficient answers as to how to assess the effect of such noise and the effect of other sources of industrial noise on people.

Hence, the author [1-4] has developed a solution that uses spectral perception data in assessment of specific noise effects on people in the presence of background noise at the observer's point. The suggested approach, when compared with that of BS 4142 standard, shows in some cases large differences in results consequences. The author shows explicitly how to use the scheme of the suggested method, in order to solve similar problems. Details for the specific case of wheel-rail squeal noise by the suggested method are given in [3-4]. The suggested method is based on the following:

First is the analogy between the privacy index (PI) and the rating level to background noise (RLBN) at the complaint point [4], see Table 1.

This observation has led us to suggest a model of scaling that is extended to industrial and environmental acoustics, using an inverse analogy to the regulation ANSI S 3.5 - 1997 [7], while using the same weighting factors. The results shown in [4] and Table 1 match perfectly this analogy and the categories used in defining the likelihood of complaints.

The design for acoustic communication and privacy in halls is generally based on suitable articulation indices (AI) as defined by ASTM E 1130 [8]. The AI depend strongly on the signal to noise ratio $(S N R)$, where the deterioration in articulation is a function of masking and of its dependence on frequency, where pure tones of higher pitch are masked by tones of lower pitch, as introduced by Mayer [9]. For that reason, pink noise is regularly used for masking, to cover a wide range of frequencies, including lower ones. Fletcher [10-11] summarized masking effects in chapter 10, pp. 153-175 of the book, and articulation scores in chapter 17, pp. 318-414. On page 334, he shows a graph of articulation versus the level of received speech above threshold and the results show that for $10 \mathrm{~dB}$ above threshold the Articulation Index is about 0.2 and for $55-60 \mathrm{~dB}$ above threshold the articulation index is about 0.9 .

Table 1: Privacy, rating noise effect, privacy PI and RLBN.

\begin{tabular}{cccc}
\hline Privacy & Rating noise effect & PI & RLBN level \\
\hline None & Intruding & $0-60$ & $>0$ \\
Poor & Exists & $60-80$ & $-3 \mathrm{~dB}$ \\
Normal & Fair & $80-95$ & $-6 \mathrm{~dB}$ \\
Good & Low & $80-95$ & $-9 \mathrm{~dB}$ \\
Extraordinary & None & $95-100$ & $-12 \mathrm{~dB}$ \\
\hline
\end{tabular}


The AI analysis is based on third-octave analysis. The key of this method is the signal to noise ratio $-S N R_{i}$ for the $\mathrm{i}^{\text {th }}$ frequency band $(200-5000 \mathrm{~Hz})$ and the corresponding weighting factors $-W_{i}$ (see Table 1 column 2 in ref. [4]) that are used to adjust speech data. The Privacy Index $(P I)$ was defined as:

$$
P I=(1-A I) \times 100 \%
$$

and

$$
P I=100\left\{1-\sum_{200}^{5000} W_{i} \times S N R_{i}\right\}
$$

For environmental acoustics we change PI to SPI (specific source ratio) and replace SNR by rating level to background noise $(R L B N)$ at the complaint point, in analogy to regulation ANSI S $3.5-1997$ [7]:

$$
S P I=100\left\{1-\sum_{200}^{5000} W_{i} \times R L B N_{i}\right\}
$$

The weighting factors, $W_{i}$, are given in the ASTM E 1130-02 [8] and column 2 in Table 1 of ref. [4] for both room and environmental acoustics, see Table 2. The numbers in Table 2 have to do with the biological mechanism of hearing and ear response at various frequencies [2].

Some calculations:

To find the RLBN at $160 \mathrm{~Hz}$ we first subtract $\mathrm{L}_{\mathrm{A} 90}$ from background noise $\mathrm{L}_{\text {AeqT }}$ and get specific sound level $\mathrm{L}_{\mathrm{s}}=\mathrm{L}_{\mathrm{ArTr}}[4,5]$. Then:

$$
\begin{gathered}
L_{s}=10 \times \lg _{10}\left[10^{0.1 \times L_{A e q, T}}-10^{0.1 \times L_{A 90, T}}\right] \\
L_{s}=10 \times \lg _{10}\left[10^{0.1 \times 66.1}-10^{0.1 \times 58.2}\right]=65.3 \mathrm{dBA}
\end{gathered}
$$

Table 2: Effect of noise, specific noise index SPI and RLBN.

\begin{tabular}{cccc}
\hline Specific noise & Rating noise effect & SPI* & RLBN** \\
\hline None & Complaints are Unlikely & $0-60 * * *$ & $<0$ \\
Low & Marginal effects & $60-80^{* * *}$ & $3 \mathrm{~dB}$ \\
High & Complaints are likely & $80-95 * * *$ & $6 \mathrm{~dB}$ \\
Very high & Complaints are most likely & $80-95 * * *$ & $9 \mathrm{~dB}$ \\
Extraordinary & Intruding, involves & $95-100$ & $12 \mathrm{~dB}$ \\
& conflicts & \\
\hline
\end{tabular}

* An example follows.

** RLBN levels are taken into account in the frequency domains, where the intruding noise is dominant above background noise (positive values), and with ignoring values at domains where the positive values are not significant.

$* * *$ Needs further research. 
Now we subtract the existing background noise level from $L_{s}$ and get $R L B N$ by using the conventional BS4142 approach up to date, for about 50 years:

$$
R L B N=65.3-58.2=7.1 \mathrm{dBA}
$$

We see from Table 1 in [4] that the squeal noise is dominant at the frequency domain $2000-5000 \mathrm{~Hz}$ and add all the $R L B N \times W_{i}$ at that frequency domain, to get the excessive noise in that frequency to get $S P I$ :

$$
\begin{gathered}
R L B N_{x} \times W_{i}=0.046+0.0286+0.0914+0.0271+0.0326=0.2256 \\
S P I=100(1-0.2256)=0.7744 \times 100=77.44 \%
\end{gathered}
$$

Then the difference between the specific noise and the background noise becomes:

$$
\begin{gathered}
R L B N_{2000-5000 \mathrm{~Hz} 6}=10 \lg _{10}\left\{10^{1.21}+10^{0.82}+10^{2.69}+10^{1.13}+10^{1.63}\right\} \\
\operatorname{RLBN}_{2000-5000 \mathrm{HZ}}=27.5 \mathrm{dBA}
\end{gathered}
$$

Thus, a huge difference between the BS4142 result (13.9 dB) and the suggested method $(27.5 \mathrm{~dB})$ is obtained - See Table 1 in [4]. This difference is likely to cause severe complaints about squeal noise. There are many combinations of calculations, and theory might be improved, but we find the suggested approach is the right way to find the subjective difference between squeal noise and background noise in each case.

The above example shows that BS 4142 is either over-simplified or at least unsatisfactory in considering the real effect of noise on people and we have tried to present an alternative method.

\section{THE USE OF THE SIMPLEX APPROACH IN ANALYSIS OF SEMI-INFINITE AND FINITE SOUND BARRIERS}

\subsection{About finite and infinite sound barriers}

The use of a finite sound barrier is a possible solution especially along intercity roads and railways, where most of the highway length passes near rural areas. While usually buildings' height in the vicinity does not exceed two floors, it is necessary to protect residents near the local segments of the lanes.

Thus, planning finite sound barriers (sometimes combined with specific topographic conditions) can be useful locally even when covering a relatively small horizontal view angle $\alpha$, yielding the necessary noise reduction and acoustic protection, while the costly 'infinite' barrier that covers 180 degrees is not needed along most of its length. It is possible to find the effect of the barrier's view angle $\alpha$ on the amount of noise reduction by using the geometrical data of the problem - see Fig. 2. Sound barriers design applies the physics of sound diffraction radiated from a source towards a chosen control point, around barrier edges over a semi-infinite domain. Maekawa [13] backed his theory by experiments, Kirchhoff's diffraction solution and added modifications. The result involved only geometry and Fresnel number. 


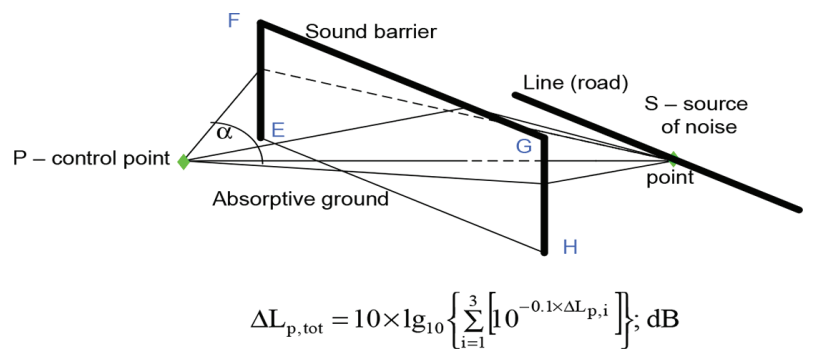

Figure 2: Finite sound barrier, a point source and an observation point.

\subsection{Introduction of the simplex method to calculation of sound barriers}

Solving insertion loss (IL) caused by an infinite barrier necessitates location of a source point, control point and a point at the top of the barrier and a vertical plane that includes the sight line between the source and the control point. The three points constitute vertices of a triangle with dependent lengths and angles. Three of them are given and three are calculated. If the three sides of the triangle are measured, one can find its angles by calculation (in any case - three unknowns out of six variables). Different angles appear in solving IL for a finite sound barrier of vertical sides. Given the triangle sides lengths, the angles are calculated from the control point to the two ends of the finite barrier by introducing Heron's formula [12], 2009. It is needed, since the barrier's location, dimensions and distances to the receiver and sources are given usually as linear dimensions and not directly by the angle $\alpha$. The same can be done with the shadow zone angle. We show here (in sections 3.3 and 3.4), that this approach is useful in other environmental acoustics problems where sound barriers are involved.

\subsection{The use of simplex theory in calculating insertion loss of sound barriers}

We expanded here the solution method using the trigonometric properties of the triangle and the n-simplex which is 2-simplex for a triangle (following Maekawa's mode [13] and Fig. 3) and irregular convex quadrilateral forms for calculation of wide barriers (following Pierce's model [14]), as well as definition of shadow zones and other problems. Such shapes can apply vector analysis to define the desired unknowns from the given data.

The trigonometric simplification of the acoustic and optical theory of diffraction enables the use of triangles to analyze the insertion loss of straight finite and semi-infinite noise barriers in 2-D and 3-D problems, see Fig. 1. Nevertheless, regularly only three of the six data that define the triangle are applied - there are three sides and three angles in each triangle and any three of them can define the others as well. The use of any of the sides and angles of the triangle can be useful in finding the effectivity of the sound barriers - for example, the shadow zone angle. Triangles and tetrahedrons are forms of simplices, defined as a finite collection of affinely independent vertices. The $n$ simplex is a convex hull of a set of $n+1$ such points in an Euclidean space of dimension $n$ or more. Examples of simplices are shown in Fig. 4.

The simplex method solves problems in linear programming. It is highly mathematical and historically it was developed by Clifford (1845-1879) [15], describing the triangle and the tetrahedron as simplest forms of confine of area and volume. It is one of the most influential algorithms. The mathematician Pieter Hendrik Schoute (1846-1923) coined the word simplex in 1902 (written by him in Latin as 'simplest'). Dantzig (1914-2005) [16] created the simplex algorithm for linear programming that solves problems for many applications in science, engineering and the arts. 

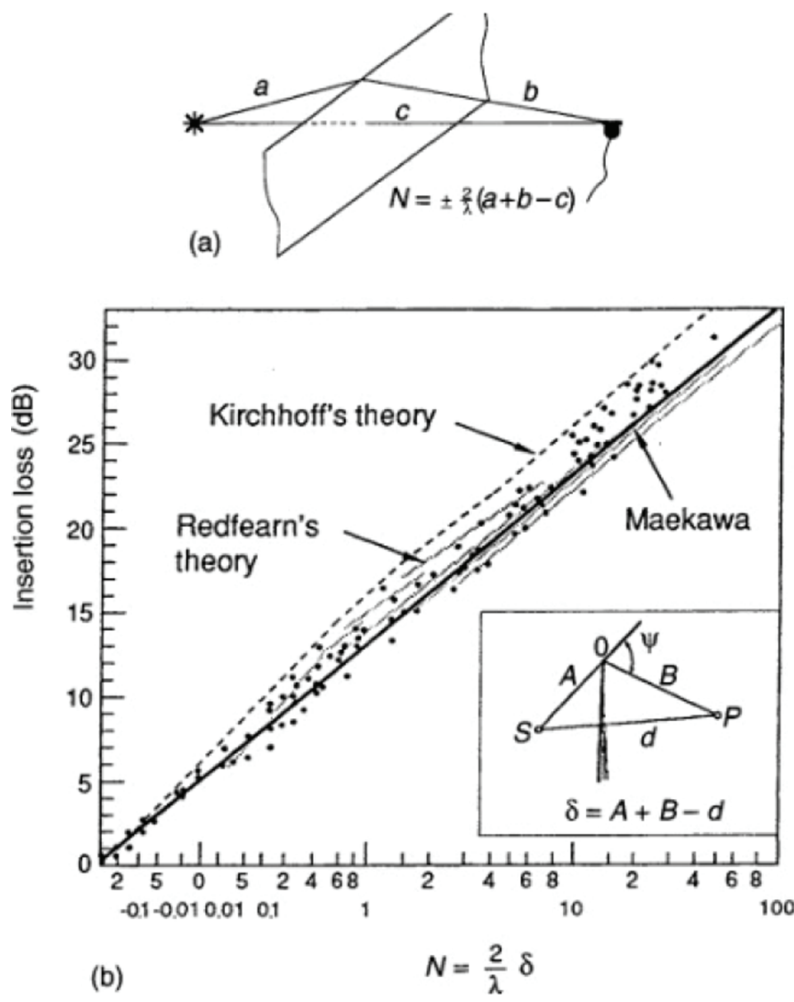

Figure 3: Insertion loss by a thin semi-infinite sound barrier using Kirchhoff's theory, Redfearn's theory, and Maekawa's formula and experiments.

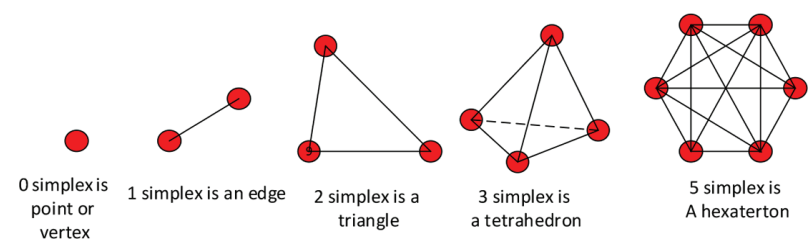

Figure 4: Examples of simplices.

3.4 Pierce's insertion loss analysis for wide barriers by irregular convex quadrilateral formulation and Maekawa's approach

Two different methods for estimating IL were published in the literature: that of Maekawa [13] and that of Pierce [14], with difference in $\Delta x-$ see eqn (5) and Fig. 3:

Maekawa's Formula reads:

$$
\begin{aligned}
& \Delta L_{p}=\left\{\begin{array}{lll}
10 \lg _{10}(3+20 N) & \text { for } & N>-0.05 \\
0 & \text { for } & N<-0.05
\end{array} ; \quad N=\frac{2 \Delta x}{\lambda} ;\right. \\
& \Delta x=r_{s}+r_{r}-R .
\end{aligned}
$$


where $N$ - Fresnel number, $P$ - control point, $R$ - the length of the sight line, SP, $r_{\mathrm{s}}-$ the distance between the source and the top of the barrier, 2-D analysis, $\mathrm{m} ; r_{\mathrm{r}}$ - the distance between the control point and the top of the barrier, 2-D analysis, $\mathrm{m} ; S$ - sound source point, $\lambda$ - source sound wave length, $\mathrm{m} ; \Delta L_{p}$ - reduction of sound level by the screen, at the control point, dBA, see Figs. 3 \& 5 .

$$
\begin{gathered}
\Delta x(\text { Maekawa })=\overline{S_{1} B_{1}^{\prime}}+\overline{B_{1} P}-\overline{S_{1} P} \\
\Delta x(\text { Pierce })=\overline{S_{1} B_{2}}+\overline{B_{1} B_{2}}+\overline{B_{2} P}-\overline{S_{1} P}
\end{gathered}
$$

Data for Maekawa triangle:

$$
\begin{gathered}
\overline{S_{1} B_{1}^{\prime}}=5.0 m ; \overline{B_{1} P}=5.4 m ; \overline{S_{1} P}=9.00 \mathrm{~m} \\
\Delta x(\text { Maekawa })=5+5.4-9=1.4 \mathrm{~m} .
\end{gathered}
$$

For Pierce quadrilateral:

$$
\begin{gathered}
\overline{S_{1} B_{2}}=4.4 m ; \overline{B_{1} B_{2}}=1.4 m ; \overline{B_{2} P}=4.6 m ; \overline{S_{1} P}=9.00 \mathrm{~m} . \\
\Delta x(\text { Pierce })=\overline{S_{1} B_{2}}+\overline{B_{1} B_{2}}+\overline{B_{2} P}-\overline{S_{1} P}=4.4+1.4+4.6-9=1.1 \mathrm{~m} .
\end{gathered}
$$

Now, for calculation of the insertion loss (IL) of the barrier of 'infinite length' eqn. (5) can be used, which for $\lambda=1 \mathrm{~m}$ is: $I L=-10 \lg _{10}(3+40 \Delta x)$. Thus:

$$
I L(\text { Maekawa })=-17.7 d B ; I L(\text { Pierce })=-16.3 d B,
$$

and the difference between the two becomes $-1.4 \mathrm{~dB}$. This difference might be much higher if each of the source $\mathrm{S}$ and the control $\mathrm{P}$ will be closer to the wall. All the difference, $\Delta(\Delta x)=\overline{B_{1} B}+\overline{B_{2} B}-\overline{B_{2} B_{1}}$, between Maekawa and Pierce models is in the triangle, $\mathrm{B}_{1} \mathrm{~B}_{2} \mathrm{~B}$. If the distance between $\mathrm{S}$ and the barrier and $\mathrm{P}$ and the barrier $\gg \lambda$, the difference is small and so is the difference in IL. If those distances tend to zero, the difference tends to infinity. In the last case, Pierce's formulation is the more logical one.

A more complicated example is shown in [12].

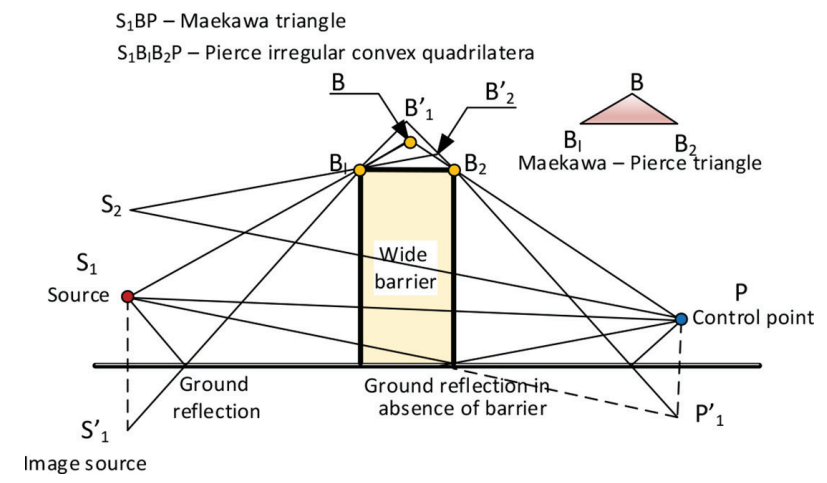

Figure 5: Maekawa - Pierce triangle. 


\section{FROM PRIMARY NUMBERS TO QRD}

Motto: Leopold Kronecker (7 December 1823 - 29 December 1891) was a German mathematician who worked on number theory and algebra. He was quoted by Weber (1893) as having said, "Die ganzen Zahlen hat der lieber Gott gemacht, alles andere ist Menschenwerk" (traditionally rendered: "God made natural numbers; all else is the work of man"; note that he says "integers", not "natürlichen Zahlen".

\subsection{Observation of scattering in nature}

Leonardo da Vinci perceived, around 1500 CE, scattering of sunlight by wood smoke, against dark background. Rayleigh confirmed this effect theoretically in 1871, and it was termed 'Rayleigh scattering'. John Tyndall discovered in 1869 particle scattering in a solution, which makes a light beam visible from the side. He suggested that the blue color of the sky is due to more scattering of the shorter wavelength of blue light by the colloidal particles of dust and water vapor in the atmosphere, beyond which the space is black. This effect is the 'Tyndall scattering'.

Specular reflections and scattering occur in nature and can be artificial. In addition, prime numbers can be found in nature. As an example of prime numbers in nature, consider the insect Magicicada that avoids meeting many of its predators (such as mammals, birds etc.) when they (the Magicicada) emerge in large numbers. A meeting with the predators could in turn lead to their decimation. However, Magicicada predators have 2 or 4 years hunting cycles, whereas the Magicicada use periods of 13 and 17 years' emergence cycles (prime numbers), which largely cancels the danger of their extinction. Prime numbers can be used also for effective design of a certain type of room acoustic diffusers.

\subsection{Application of absorption, reflections and scattering in interiors acoustics}

Two acoustic methods for weakening disturbing echoes in rooms are available:

One way is by converting the sound energy into a different kind of energy - usually heat. It means acoustic energy absorption. However, such a change might involve the cost of losing part of the desired sound level - say, in the hall.

The second method is based on 'breaking' specular and resonant reflections into distributed radiation, as uniform as possible, and lower amplitudes. It is defined as acoustic scattering.

As an example of interiors acoustics, scatterers are used to diffuse specular reflections. Specular reflections, combined with interiors and wall shaping, are used to form better acoustics for audience and visitors, see Fig. 6. Yet, they are less efficient than mathematically designed scatterers. The result of such scatterers is that instead of an echo, the interior can become reverberant and of higher quality.

The special kind of scatterers illustrated here is based on principles of the mathematical theory of numbers - see for example, Hardy and Wright [17]. The practical outcome of this theory and application is a variety of products and many patents. Still, there is much room for better physical modelling and engineering innovations.

\subsection{Prime numbers, residues and quadratic residues}

\subsubsection{Prime numbers - basic definition.}

A prime number is a natural number greater than 1 that has no positive divisions other than 1 and itself. 


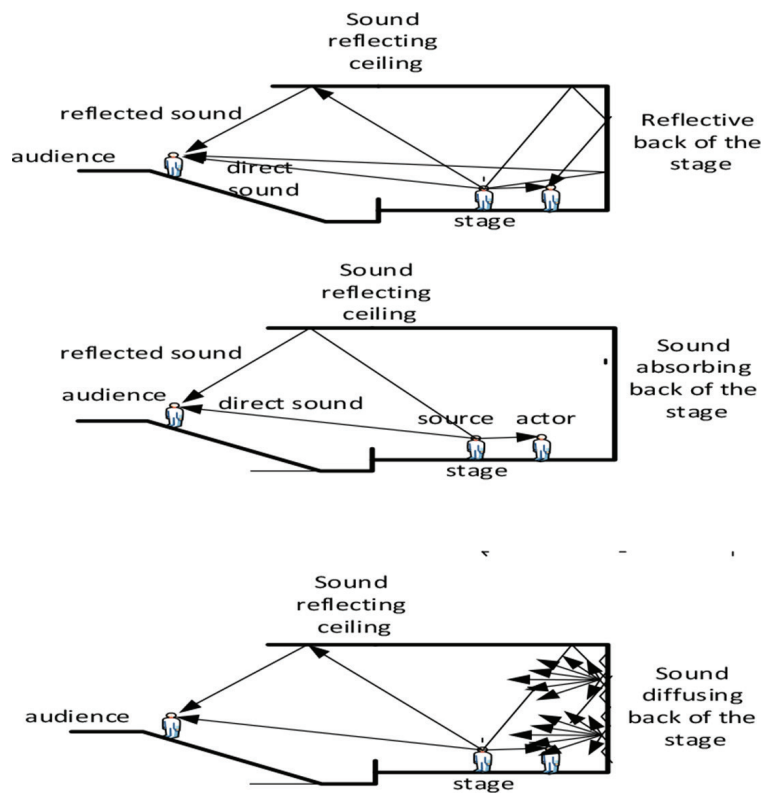

Figure 6: Specular reflection from the back wall of a stage (top), an absorbent back wall of a stage (middle) and scattering back wall of a stage (bottom).

Some notes: (1) Zero is not a primary number. (2) The number 1 is not a primary number in spite of the fact that the definition suits the number. It is because this number is counted twice. (3) The only even prime is 2 , since all the other even numbers are multiplied by 2 .

\subsubsection{Euclid's contribution}

Euclid of Alexandria (about $325 \mathrm{BC}$ to about $265 \mathrm{BC}$ ) residues:

Theorem: The number of primes is infinite.

If we assume that there are $\mathrm{p}_{1}, \mathrm{p}_{2}, \mathrm{p}_{3}, \ldots \mathrm{p}_{\mathrm{n}}$ primes, then the number $\mathrm{N}=\mathrm{p}_{1} \cdot \mathrm{p}_{2} \cdot \mathrm{p}_{3} \cdot \ldots \cdot \mathrm{p}_{\mathrm{n}} \cdot 1$ is not divisible by any of the existing primes. Thus $\mathrm{N}$ is either a new prime number, or a product of two new primes so that it can be written in the form of the base theorem:

The base theorem of the number theory: Every number that differs from $0,1,-1$ can be written as a product of irreducible numbers. This factorization is unique, regardless of the numbers $1,-1$ and the rank of the factors. But this is only possible if either $\mathrm{N}$ is a new prime, or it is a product that includes a new prime.

Euclidean Algorithm: Dividend equals divisor times quotient remainder (R).

$\mathrm{R}=\mathrm{n}^{2} \bmod (\mathrm{N})=\mathrm{n}^{2} \operatorname{modulu}(\mathrm{N})=$ Remainder in a maximum length sequence. As an example, the quadrature residue $15^{2} \bmod (13)$ equals $\mathrm{R}(4)$, see Table 3.

\subsubsection{Gauss' contribution}

In the 18th century, Gauss discovered the quadratic residuals and quadratic reciprocity as a pure mathematical issue and the link to acoustic diffusers came later through physics.

\subsection{The basic contribution of physics}

The basic contribution of physics to the design of acoustic diffusers begun with X-ray research. In 1895 Wilhelm Röntgen discovered X-rays, which could pass through his 
Table 3: Quadratic residues.

\begin{tabular}{|c|c|c|c|c|c|c|c|c|c|c|c|c|c|c|c|c|c|c|c|}
\hline n & 1 & 2 & 3 & 4 & 5 & 6 & 7 & 8 & 9 & 10 & 11 & 12 & 13 & 14 & 15 & 16 & 17 & 18 & 19 \\
\hline$n^{2}$ & 1 & 4 & 9 & 16 & 25 & 36 & 49 & 64 & 81 & 100 & 121 & 144 & 169 & 196 & 225 & 256 & 289 & 324 & 361 \\
\hline $\bmod 3$ & 1 & 1 & 0 & 1 & 1 & 0 & 1 & 1 & 0 & 1 & 1 & 0 & 1 & 1 & 0 & 1 & 1 & 0 & 1 \\
\hline $\bmod 5$ & 1 & 4 & 4 & 1 & 0 & 1 & 4 & 4 & 1 & 0 & 1 & 4 & 4 & 1 & 0 & 1 & 4 & 4 & 1 \\
\hline $\bmod 7$ & 1 & 4 & 2 & 2 & 4 & 1 & 0 & 1 & 4 & 2 & 2 & 4 & 1 & 0 & 1 & 4 & 2 & 2 & 4 \\
\hline $\bmod 11$ & 1 & 4 & 9 & 5 & 3 & 3 & 5 & 9 & 4 & 1 & 0 & 1 & 4 & 9 & 5 & 3 & 3 & 5 & 9 \\
\hline $\bmod 13$ & 1 & 4 & 9 & 3 & 12 & 10 & 10 & 12 & 3 & 9 & 4 & 1 & 0 & 1 & 4 & 9 & 3 & 12 & 10 \\
\hline $\bmod 17$ & 1 & 4 & 9 & 16 & 8 & 2 & 15 & 13 & 13 & 15 & 2 & 8 & 16 & 9 & 4 & 1 & 0 & 1 & 4 \\
\hline $\bmod 19$ & 1 & 4 & 9 & 16 & 6 & 17 & 11 & 7 & 5 & 5 & 7 & 11 & 17 & 6 & 16 & 9 & 4 & 1 & 0 \\
\hline
\end{tabular}

hand, casting the bone shadow on a fluorescent screen. In 1901, he won the Nobel Prize for this.

Von Laue (1879-1960) discovered the diffraction of X-rays by crystals as the starting point to crystallography. For this, he won the Nobel Prize in 1914.

William Henry Bragg (1862-1942) and William Lawrence Bragg (1890-1971) determined crystals structure by use of x-ray diffraction. Their law shows the wavelength relation to the angle of constructive diffraction of reflections. Both of them were Nobel Prize laureates in 1915, see [25-27].

Some x-ray applications today include crystallography, biotechnology, forensic science, DNA double helix, industrial nondestructive testing (NDT), and Medicine.

\subsection{Schroeder's breakthrough and the QRD}

Various types of surfaces are used in general as sound diffusers, and there is no single specific solution. An example is a concave surface that radiates sound in directions that differ from mirror reflections. In this paper, we show one type of diffusers - the QRD. These diffusers are based on wave interference because of reflections sequence from surfaces of different distances, yielding phase differences. The mathematical basis, which explains the response of such surfaces and the shape of different reflections, is based on the method shown by Schroeder and applied to acoustics of halls and other interiors.

QRD is a sequence of periodic grooves (slits, wells) of different depths, $d(x)$, but equal width, w, separated by thin, rigid partitions. $d(x)$ was refined by Schroeder, using mathematical sequences.

A common use of QRD is in the design of 'an ideal' concert hall of smooth reverberation response, by weakening resonance, specular and standing waves effects. Theoretically, such a response at all frequencies for a typical geometry of a hall is impossible. However, it is possible to improve its reverberation response by different methods. Of special importance is a well-diffused and uniform sound field within the hall and in the satisfaction of all the audiences. This is just as daylight: it is not a specular reflection typical to mirror reflection or a narrow scattering products.

Quadratic residue diffusers (QRD) are a certain type of scatterers that distributes the specular wave that hits a rough surface in a very wide angle (when the angle is narrow, and centers along the specular reflection line, it is defined as a scattered wave). Both diffusion and 
scattering appear both in nature and intentional design. It is in contrast to specular reflected (acoustic) energy from a plane, smooth surface, in a narrow beam.

During the $70 \mathrm{~s}$, Schroeder proposed a design method for surfaces that can diffuse efficiently reflections of specular waves, based on sequences of maximal length (Schroeder [18]) and sequences of quadratic residues diffusers (Schroeder [19]). He found that by introducing phase shift to the sound that is reflected from certain regions of the scatterer's surface, an ideal diffuser will be obtained. This phase shift is defined as the reflection factor.

Such panels have been applied in the design of concert halls and recording studios, as a first experiment on phase grating scatterers.

A scatter diagram from a surface with reflection coefficients alternating in $\mathrm{x}$ direction is according to one period of maximum-length sequence such as $(-++-+-++++---+-)$ (see Fig. 1, ref. [19]). Such results were obtained in model experiments with electromagnetic microwaves.

4.5.1 An estimate of the Schroeder diffuser

This is obtained using

$$
\text { Well Depth }=n^{2} \bmod N \frac{\lambda}{2 N}
$$

Let $n$ be the well number $(1,2,3, \ldots) . N$-a primary number that defines the total number of wells $(5,11,13, \ldots), \lambda$ - the design wavelength. With such data, Schroeder diffusers have an effective bandwidth that ranges from half an octave below $f_{\mathrm{o}}$ up to the frequency defined by the width of the wells. Schroeder recommended the width of the well to be the design wavelength multiplied by $0.137 \lambda_{\mathrm{o}}$.

The efficiency of the Schroeder diffuser is much improved, if each well is isolated from its neighbors by a thin partition. However, such diffusers have a lower frequency limit, below which they are practically ineffective. This limit is defined as the Schroeder frequency.

This kind of diffusers revolutionized the diffusion quality that could be achieved, leading use them more in modern concert halls. Two examples are the Carnegie Hall in NY and Michael Fowler Centre in New Zealand.

\subsubsection{A calculated example}

The sequence of depths is defined from the sequence of quadrature residues $(\mathrm{QR})$, for the maximum length sequence, MLS. The sequence is mathematical, and it is based on the prime number, $N$, say, $N=13$. The $N$ th term is given by $n^{2} \bmod N$.

For example, see the following QRD calculation of the work frequency:

For a sequence of quadratic residuals $s_{\mathrm{n}}$, the depth of the well is:

$$
d_{n}=\frac{s_{n} \lambda_{0}}{2 N}=\frac{s_{n} c}{2 N f_{0}} \quad \lambda_{0}=c T_{0} \frac{c}{f_{0}} ; d_{n}=\frac{12 \times 340}{2 \times 13 \times f_{0}} . \text { If } \mathrm{d}_{\mathrm{n}}=150 \mathrm{~mm},
$$

In addition, the work frequency is: $f_{0}=\frac{12 \times 340}{2 \times 13 \times 0.15}=\frac{4080}{3.9}=1046 \mathrm{~Hz}$

4.5.3 Review of some theoretical aspects of reflections from rough surfaces and QRD Problems of scattering and diffusion involve integrations, basically, and the break-through paper by Schroeder [18] belongs to this mathematical approach, as well as others that deal with scattering and diffusion from rough surfaces. See also regulations ISO 17497-1-2004 
[21] and ISO 17497-2:2012 [22] for sound scattering in rooms that are important in this context.

Schroeder [18] summarized the relation of the spatial relation between the spatial distribution of reflected coefficient $r(\mathrm{x})$ along a wall and its scattering amplitude $S(\alpha)$ : $S(\alpha)=\int\left\{r(x) \exp \left[2 \pi j x\left(\sin \alpha-\sin \alpha_{1}\right) / \lambda\right]\right\} d x$, and $\alpha$ as the scattering angle with respect to the normal to the wall. $\alpha_{\mathrm{i}}$ is the angle of incidence and $\lambda$ is the wave length of the incident wave. Thus, $S(\alpha)$ is the Fourier transform of the reflection coefficient, $r(\mathrm{x})$, along the wall, but with a change by a variable. Then, the relation between $\alpha$ and the wave number, $k$, becomes:

$$
k=2 \pi\left(\sin \alpha-\sin \alpha_{1}\right) / \lambda, \text { or its inverse. }
$$

For normal incidence, $\alpha_{\mathrm{i}}=0$, and scattered energy up to $\alpha_{\max }= \pm 90^{0}$, the highest $k$ of $r(x)$ Fourier transform becomes: $k_{\max }=2 \pi / \lambda$. If the reflection coefficient $r(x)$ is designed according to the max-length sequence $\sigma_{n}$, then $r(x)=\sum_{n=-\infty}^{\infty}\left\{\sigma_{n}\right.$ rect $\left.\left(\frac{x}{d}-n\right)\right\}$, the rect function equals 1 between \pm 0.5 and zero elsewhere. The power spectrum of $\mathrm{r}(\mathrm{x})$ is:

$$
\left|R\left(k=\frac{2 \pi l}{N d}\right)\right|^{2}=\left(\frac{N+1}{N^{2}}\right)[\sin (\pi l / N) / \pi l / N]^{2} \text {. }
$$

$l$ is any integer but zero or multiples of the sequence length, $N$. For normal incidence: $l=N \sin \left(\alpha_{i} d\right) / \lambda$, thus, the energy only in discrete directions is: $\alpha_{i}=\arcsin (\lambda l) / N d$. Now, in terms of intensities:

$$
\left|S\left(\alpha_{i}\right)\right|^{2}=\left(\frac{N+1}{N^{2}}\right)\left[\sin \left(\pi \sin \alpha_{i} d / \lambda\right) / \pi \sin \alpha_{i} d / \lambda\right]^{2}
$$

\subsubsection{Schroeder frequency [18] - The crossover frequency}

The crossover frequency, or Schroeder frequency, is the transition of lower frequency that room behavior is allowing special application in room acoustics. Schroeder frequency [23, 24] is approximately:

$$
f_{c}=\sqrt{ }\left(\frac{T}{V}\right)
$$

where $V$ is the volume of the room $\left(\mathrm{m}^{3}\right), T$ is the reverberation time (s).

\subsection{Planning hypotheses based on the design equation of the QRD}

The design equation of QRD results in maximum diffusion at the design frequency, $f_{\mathrm{o}}$ and wavelength, $\lambda_{\mathrm{o}}$. The maximum diffusion is also obtained at multiples of $f_{\mathrm{o}}$ by integer numbers, $n: n f_{\mathrm{o}} . f_{\mathrm{o}}$ is often chosen as the lowest frequency, below which the diffuser is not efficient.

Following interference theory, if the wells of the QRD are very flat, the roughness of the surface area almost diminishes as compared with $\lambda: \lambda / 2>>d$ and the wave will not be affected by the roughness - which eventually ends with specular reflection. If the width of the wells is very narrow, the wave skips the grooves. 
The low cutoff frequency is often chosen as: $\lambda / 2=d_{\max }$, but actually, the limit frequency is lower by about one octave from this value. Yet, $d_{\max }$ depends on the room dimensions. The side lobes of the acoustic radiation are crucial in periodic diffusers, since they increase the diffusion, except where the diffuser's period is larger, than $\lambda$, and no side lobes appear.

The condition for upper frequency limit is that $<\frac{\lambda}{2}$, but in spite of the fact that the design presents this limit of plane wave, the limit functions also above this region. Too narrow wells are difficult to perform and they are involved with viscous damping and boundary layers. Thus, the width of the well is practically 2.5 to $5 \mathrm{~cm}$. An example of design of a 2-D scatterer, $S_{n, m}=\left(r^{n}+r^{m}\right)$ is given in [20].

\section{SUMMARY}

Many areas in acoustic engineering design methods apply calculations and standards that yield simplified formulation. However, in many cases, this approach is over-simplified.

Thus, the current paper introduced three cases. The author has originally published the first two, and the third one scans about 2130 years of top mathematical and physical development, with the final link to acoustic technology in 1975, which has created a commercial product.

The bottom line concluded in the present paper is that basic science supports all existing and future technologies, and when investment in science is either diminished or stopped, the quality of engineering R\&D regresses and deteriorates. Sometimes it is useful to dig ancient observations and theoretical ideas from the past in order to improve modern technologies.

\section{REFERENCES}

[1] Rosenhouse, G., The acoustical comfort and speech privacy in the design of flexible open-plan offices. 42th Inter-noise Conference, Innsbruck, 15-18 September, 2013, presentation \#140, 2013.

[2] Rosenhouse, G., The spectral effect of masking of intruding noise by environment background noise. 167th Meeting of the Acoustical Society of America, Providence, Rhode Island, 5-9 May, 2014.

[3] Rosenhouse, G., The spectral effect of masking of intruding squeal noise by environmental background noise - A detailed analysis. Spring 2016 Meeting of the Acoustical Society of America, Salt Lake City, Utah, 23-27 May, 2016.

[4] Rosenhouse, G., The subjective analysis of wheel-rail squealing noise by modification of the British standard BS4142:2014, Inter Noise 2018, Chicago, Illinois, 26-29 August 2018, Presentation 1887 (On USB Device), 2016.

[5] BS4142:1997, Method for Rating Industrial Noise Affecting Mixed Residential Areas and Industrial Sites, British Standards Institution, BSI, London, 1997 and BS4142:2014, Method for Rating and Assessing Industrial and commercial sound, British Standards Institution, BSI, London, 2014.

[6] ISO 1996, Assessment of Noise with Respect to Community Response -International Organization of Standardization, Geneva, 1971; (see also 1982-1987, 1998).

[7] ANSI S3.5-1997, Methods for calculation of the speech intelligibility Index, American National Standard, Standards Secretariat, Acoustical Society of America, NY, 1997

[8] ASTM E 1130 - 02, Standard Classification for Determination of Articulation Class, American Society for Testing and Materials, 2001.

[9] Mayer, A.M., Researches in acoustics, Philosophical Magazine, 2, pp. 500-507, 1876. https://doi.org/10.1080/14786447608639144 
[10] Fletcher, H., Auditory patterns, Reviews of Modern Physics, 12, pp. 47-65, 1940. https://doi.org/10.1103/revmodphys. 12.47

[11] Fletcher, H., Speech and Hearing in Communication, ASA, NY, pp. 153-175, 318-414, 1995. Originally published by van Nostrand, NY 1953.

[12] Rosenhouse, G., Finite sound barriers and the use of Heron's formula. INTER-NOISE 2009, Ottawa, Canada, August 23-26, 2009. See also, G.Rosenhouse, The use of the simplex approach for analysis of infinite and finite sound barriers. Inter-Noise 2019, Madrid, Spain, 16-19.July, 2019, Presentation 1555 (On USB Device)

[13] Maekawa, Z-I., Noise reduction by screens. Journal of Applied Acoustics, 1, pp. 157-173, 1968. https://doi.org/10.1016/0003-682x(68)90020-0

[14] Pierce, A.D., Diffraction of sound around corners and over wide barriers. Journal of the Acoustical Society of America, 55(5), pp. 941-955, 1974. https://doi.org/ $10.1121 / 1.1914668$

[15] Hitzer, E., Introduction to Clifford's geometric algebra. SICE Journal of Control, Measurements and System Integration, 4(1), pp. 1-11, 2011.

[16] Dantzig, G.B., Origins of the Simplex Method, Technical Report, Stanford University Systems Optimization Lab, 87-5, 1987.

[17] Hardy, G.H. \& Wright, E.M., (Reviewed by: D.R., Heath-Brown, D.R., Silverman, J.H., Wiles, A.), An Introduction to the Theory of Numbers, 6th ed. Oxford University Press, NY, USA, 2008.

[18] Schroeder, M.R., Diffuse sound refection by maximum length sequences. Journal of the Acoustical Society of America, 57, pp. 149-150, 1975. https://doi.org/10.1121/1.380425

[19] Schroeder, M.R., Binaural dissimilarity and optimum ceilings for concert halls: More lateral sound diffusion. Journal of the Acoustical Society of America, 65(4), pp. 958-963, 1979. https://doi.org/10.1121/1.382601

[20] Cox, T. \& D’Antonio, P., Acoustic Absorbers and Diffusers: Theory, Design and Application, Spon Press: London and New York, 2004; Tailor \& Francis, 2nd ed. 2009.

[21] ISO 17497-1:2004 - Acoustics - Sound scattering properties of surfaces. Part 1: Measurement of the random - incidence scattering coefficients in a reverberation room.

[22] ISO 17497-2:2012 - Acoustics - Sound scattering properties of surfaces. Part 2: Measurement of the directional diffusion coefficient in a free field.

[23] Schroeder, M.R., Die statistischen Parameter der Frequenzkurve von großen Räumen. Acustica, 4, pp. 594-600, 1954. [English translation: M. R. Schroeder, Statistical parameters of the frequency response curves of large rooms. Journal of Audio Engineering Society, 35, pp. 299-306, 1987. Note: In the original 1954 paper a more conservative factor of 4000 was proposed instead of 2000 in Eq. corresponding to a tenfold mod.

[24] Schroeder, M.R., Sequences from number theory for physics, signal processing and Art. Acoustical Physics, 49(1), pp. 97-108, 2003. https://doi.org/10.1134/1.1537395

[25] Bragg, W.H., X rays and crystals. Nature, 90, p. 219, 1912. https://doi.org/ 10.1038/090219a0

[26] Bragg, W.H., X rays and crystals. Nature, 90, pp. 360-361, 1912. https://doi.org/ 10.1038/090360d0

[27] Bragg, W.L., The diffraction of short electromagnetic waves by a crystal. Proceedings of the Cambridge Philosophical Society, XVII, 1913. 